\title{
Abastecimiento de alimentos producidos por la agricultura familiar en la alimentación escolar en Costa Rica: Potencialidades y limitaciones
}

\author{
Marianela Zúñiga-Escobar \\ Facultad de Medicina, Universidad de Costa Rica - San José - Costa Rica \\ Universidade Federal de Rio Grande do Sul - Porto Alegre - RS - Brasil \\ ORCID: https://orcid.org/0000-0002-6688-3665

\section{Catia Grisa} \\ Universidade Federal de Rio Grande do Sul - Porto Alegre - RS - Brasil \\ ORCID: https://orcid.org/0000-0001-6685-4875 \\ Gabriela Coelho-de-Souza \\ Universidade Federal de Rio Grande do Sul - Porto Alegre - RS - Brasil \\ ORCID: https://orcid.org/0000-0002-7652-9475
}

\section{Resumen}

La alimentación escolar ha sido considerada como un mecanismo de reconexión entre la producción de la agricultura familiar y el consumo de alimentos, siendo un punto de encuentro entre la alimentación pública y el desarrollo sostenible. En ese proceso cobra interés el funcionamiento del Programa de Abastecimiento Institucional (PAI) en el ámbito del Programa de Alimentación y Nutrición del Escolar y del Adolescente (PANEA). El objetivo de este artículo es analizar las principales potencialidades y limitaciones del proceso de abastecimiento de alimentos producidos por familias agricultoras en la alimentación escolar en Costa Rica. Se realizó una investigación cualitativa con entrevistas semiestructuradas y grupos de discusión con participación de diversos actores involucrados en dos proyectos piloto (2016-2018) de la iniciativa Escuelas Sostenibles en las localidades de Frailes de Desamparados y Coto Brus. Como resultado se evidenció que las principales limitaciones radican en la falta de mecanismos que proporcionen un trato diferenciado de abastecimiento de alimentos producidos por familias agricultoras locales. Por su parte, las experiencias potenciales del proceso se expresan en valores que sobrepasan los aspectos económicos, que se presentan en manifestaciones socioculturales y éticas generadas durante el recibimiento de alimentos en los centros educativos, respondiendo a la construcción social de mercados que caracteriza a las compras públicas de alimentos.

Palavras-chave: Abastecimiento institucional. Agricultura Familiar. Alimentación escolar. Seguridad alimentaria y nutricional. Sistemas alimentarios sostenibles. 


\section{Food supply produced by family farming for school feeding in Costa Rica: Potentialities and limitations}

\section{Abstract}

School feeding has been considered as a linking mechanism between family farming production and food consumption, being a meeting point between public feeding and sustainable development. In this process, the operation of the Programa de Abastecimiento Institucional (PAI) within the scope of the Programa de Alimentación y Nutrición del Escolar $y$ del Adolescente (PANEA) regain interest. The objective of this article is to analyze the main potentialities and limitations of the food supply process produced by agricultural families for school feeding in Costa Rica. A qualitative research was carried out with semistructured interviews and discussion groups with the participation of various actors involved in two pilot projects (2016-2018) of the Escuelas Sostennibles initiative in the towns of Frailes in Desamparados and Coto Brus. As a result, it is evident that the main limitations lie in the lack of mechanisms that provide differentiated treatment for food supply produced by local agricultural families. On the other hand, the potential experiences of the process are expressed in values that exceed the economic aspects, they are presented in sociocultural and ethical manifestations generated during the reception of food in educational centers, responding to the social market construction that characterize public purchases of food.

Keywords: Institutional supply. Family farming. School feeding, Food and Nutrition Security. Sustainable food systems.

\section{Abastecimento de alimentos produzidos pela agricultura familiar na alimentação escolar na Costa Rica: Potencialidades e limitações}

\section{Resumo}

A alimentação escolar tem sido considerada como um mecanismo de reconexão entre a produção da agricultura familiar e o consumo de alimentos, sendo um ponto de encontro entre a alimentação pública e o desenvolvimento sustentável. Nesse contexto, destaca-se o funcionamento do Programa de Abastecimento Institucional (PAI) no âmbito do Programa de Alimentação e Nutrição do Escolar e do Adolescente (PANEA). Este artigo tem como objetivo analisar as principais potencialidades e limitações do processo de abastecimento de alimentos produzidos por famílias agricultoras na alimentação escolar na Costa Rica. Foi realizada uma pesquisa qualitativa com entrevistas semi-estruturadas e grupos de discussão com participação de diversos atores envolvidos na iniciativa Escolas Sustentáveis, a partir de dois projetos piloto (2016- 2018) nas localidades de Frailes de Desamparados y Coto Brus. Como resultado se evidenciou que as principais limitações centram-se na falta de mecanismos que proporcionem um tratamento diferenciado no abastecimento de alimentos produzidos por famílias agricultoras locais. Por outro lado, os potenciais se expressam nos valores que transcendem aos aspectos econômicos, que se apresentam em manifestações socioculturais e éticas geradas durante o processo abastecimento de alimentos nos centros educativos, respondendo à construção social de mercados que caracteriza as compras públicas de alimentos.

Palabras clave: Abastecimento institucional. Agricultura familiar. Alimentação escolar. Segurança alimentar e nutricional. Sistemas alimentares sustentãveis. 


\section{Introducción}

Los programas de alimentación escolar se vuelven un potencial transformador significativo en términos de sostenibilidad, al estar sumergidos en dos grandes debates que reevalúan los objetivos de la alimentación escolar. El primero de ellos es el debate de desarrollo sostenible y la "nueva ecuación alimentaria", alineándose con la emergencia de un régimen alimentario alternativo. El segundo debate se relaciona con el papel predominante que tiene el Estado para conseguir alcanzar ese nuevo régimen alimentario (APARECIDA; SCHNEIDER; VERARDI, 2015). Este tema que incluye la acción del Estado, denominado por algunos autores como "Estado Verde" o democracia ecológica (MORGAN, 2006; SONNINO et al., 2018), es en donde la alimentación escolar ha sido considerada como un punto de encuentro entre la alimentación pública y el desarrollo sostenible.

Para Sonnino, Bassinello y Lozano (2018), los programas de alimentación escolar representan un tema "viejo y nuevo" en el que sus valores y decisiones políticas, varían significativamente sus objetivos, dependiendo del contexto y de los problemas a los que se dirigen. En la actualidad, estos valores se reflejan en diversas experiencias que buscan establecer relaciones entre la alimentación escolar y el abastecimiento de alimentos producidos por agricultores locales. Entre esas experiencias está el caso del movimiento "Farms to School" del United States Department of Agriculture, ejecutado por medio del Food and Nutrition System de los Estado Unidos (NATIONAL FARM TO SCHOOL NETWORK, 2016). También el caso de Powy Public Procurment Partnership en Reino Unido, basado en el establecimiento de alimentos locales orgánicos en las escuelas y hospitales (POWY PUBLIC PROCUREMENT PARTNERSHIP, 2002).

En América Latina la experiencia del Programa Nacional de Alimentação Escolar (PNAE) de Brasil fue difundida por la iniciativa de América Latina y Caribe sin Hambre 2025, el Gobierno de Brasil (por medio del Fondo para o Desenvolvimento da Educação (FNDE) y la Organización para la Alimentación y la Agricultura (FAO por sus siglas en inglés) mediante el Proyecto de Fortalecimiento de los Programas de Alimentación Escolar. Este proceso motivó a las instancias gubernamentales de otros países para rediseñar sus programas.

En el caso de Costa Rica, la implementación de la iniciativa se denominó Escuelas Sostenibles, siendo implementado dos proyectos piloto en las localidades de Frailes de Desamparados, en la Región Central del país y otro en el Cantón de Coto Brus, en la Región Brunca. Durante la ejecución, hubo un interés especial por la problemática que representa la inserción de alimentos producidos por familias agricultoras en la alimentación escolar, debido a las implicaciones de ejecución articulada entre dos programas con amplia trayectoria en el país: el Programa de Abastecimiento Institucional (PAI) y el Programa de Alimentación y Nutrición del Escolar y del Adolescente (PANEA).

Esta forma de abastecimiento alimentario representa desafíos de gobernanza que requieren de acciones intersectoriales, con el fin de que exista un beneficio para la producción local y el consumo escolar. Por esta razón, el presente artículo tiene como objetivo presentar un análisis sobre las potencialidades y limitaciones que fueron percibidas por los actores involucrados en los proyectos piloto, durante los años 2016 al 2018, y así proporcionar una base que contribuya con mejores mecanismo de abastecimiento hacia la promoción de sistemas alimentarios sostenibles. 


\section{Metodología}

Los resultados presentados en este artículo forman parte de la tesis de doctorado del Programa de Pós-graduação em Desenvolvimento Rural da Universidade Federal de Río Grande do Sul- Brasil, titulada: "Enfoque sistémico e la seguridad alimentaria y nutricional aplicado a la gobernanza de la inserción de alimentos producidos por la agricultura familiar en la alimentación escolar". La investigación se desarrolló en el transcurso de los años 2016 y 2018, cuando se encontraban en ejecución los proyectos piloto de la Iniciativa Escuelas Sostenibles en las localidades del distrito de Frailes de Desamparados, en la Región Central y, en el cantón de Coto Brus, en la Región Brunca de Costa Rica.

Se trata de una investigación de enfoque cualitativo que constó de una etapa de recolección de la información durante dos periodos: 1) julio- agosto del año 2016, se utilizó una técnica de entrevista a profundidad dirigida a 18 informantes clave, quienes al ser personas relacionadas con la temática, tenían conocimiento del contexto y de los actores involucrados en la problemática. De 2) febrero- agosto del año 2017, se realizó la técnica de entrevista semiestructurada a 46 actores involucrados en los proyectos piloto de las localidades mencionadas. La información obtenida de las entrevistas se trianguló y complementó con los resultados de tres grupos de discusión participativa utilizando la técnica de sociograma, uno de ellos a nivel nacional, conformado por representantes del Comité Técnico Nacional (instancia creada para la coordinación intersectorial de los proyectos piloto). Los otros dos grupos se realizaron en el nivel local, en Frailes de Desamparados con participación de representantes de agricultores, miembros del Centro Agrícola Cantonal y de la escuela, y otro en Coto Brus con representantes de las organizaciones de agricultores.

Las entrevistas y grupos de discusión fueron grabadas y transcritas a formato de Word. Posteriormente, para el análisis de contenido se utilizó el software NVivo®. La información de cada entrevista fue clasificada en nodos, de acuerdo con las preguntas establecidas en la entrevista semiestructurada, y para el caso de los grupos de discusión, se realizó un análisis del contenido de cada grupo para utilizarlo de forma triangulada y complementaria.

\section{El abastecimiento de la alimentación escolar en Costa Rica}

El Programa de Alimentación y Nutrición del Escolar y del Adolescente (PANEA) tiene como objetivo ofrecer alimentación complementaria y promover hábitos alimentarios saludables entre la población estudiantil, usando este espacio para favorecer alimentos nutritivos, reforzar hábitos de higiene adecuados y hábitos de comportamiento en relación con la alimentación diaria (MEP, 2012). Para eso, la Dirección de Programas de Equidad (DPE) del Ministerio de Educación Pública (MEP) es la encargada de dictar los lineamientos técnicos, directrices y manuales de procedimientos para orientar la asignación, ejecución, supervisión y control de los recursos canalizados para financiar el PANEA (Art. 109 del Reglamento General de Juntas).

Por su parte, los encargados de la aplicación del menú en la escuela son: la Junta Escolar o Administrativa junto con el Patronato Escolar (Reforma al Reglamento de Patronatos Escolares $\mathrm{N}^{\circ}$ 38165-MEP, 2013) y el Director Institucional, quienes seleccionan el menú diario basado en el Manual de Menú (MEP, 2012). Este proceso debe ser auxiliado por el Comité de Salud y Nutrición (organismo de apoyo para promover el desarrollo de la educación en salud y nutrición, el desarrollo de huertas y proyectos productivos y para velar por el adecuado funcionamiento del comedor y la soda estudiantil), conformado por el director/a, representación docente, de padres de familia, de trabajadores del comedor y 
estudiantes de la Escuela. Una vez acordado el menú, éste debe ser firmado y sellado por la Junta Escolar o Administrativa para garantizar su aprobación (MEP, 2012).

Las Juntas de Educación y Juntas Administrativas son las que deben administrar los recursos económicos provenientes del Fondo de Desarrollo Social y Asignaciones Familiares (FODESAF), asignados al centro educativo por medio del PANEA, para el funcionamiento de los comedores escolares (Art. 45 de la Ley Fundamental de Educación). Además de la compra de alimentos, las Juntas deben administrar los fondos públicos para el financiamiento de bienes y servicios complementarios tales como: mobiliario, equipo, la contratación de personal para el comedor, así como el desarrollo de las huertas estudiantiles (Art. 10 del Reglamento General de Juntas).

La compra de alimentos es descentralizada, y según las disposiciones del PANEA, la Junta debe realizar el proceso de Contratación del proveedor de alimentos de conformidad con el proceso de licitación pública de la Ley de Contratación Administrativa № 7494 y sus reformas y, el Reglamento General de Juntas de Educación y Administrativas (MEP, 2012). Sin embargo, debido a las exigencias fiscales para justificar las compras, algunas Juntas en el país están autorizadas por la Contraloría General de la República, para hacer las compras mediante la contratación directa. Para eso, las Juntas deben solicitar la autorización a la Contraloría.

De acuerdo con la figura 1, existen tres formas de contratación permitidas para que las Juntas realicen la compra de los alimentos para los comedores escolares. Para el caso de esta investigación, las dos formas de contratación que incluyeron la inserción de alimentos producidos por familias agricultoras, corresponden: i) A la contratación directa \#1 por medio del Consejo Nacional de Producción (CNP), este es el caso del proyecto piloto en Coto Brus, en donde el CAC se encuentra adscrito como proveedor del PAI y el proceso de pedido y pago se realiza por medio del CNP. La otra forma de contratación es ii) la contratación directa \#3 que corresponde a la compra directa con la organización de agricultores, este es el caso del proyecto piloto en Frailes de Desamparados, en donde las personas de los centros educativos realizan el pedido y pago de los alimentos directamente con el CAC. En ambos casos la entrega de alimentos se realizó de forma directa, es decir, los agricultores llevan los alimentos al CAC correspondiente y desde esta entidad se distribuían los alimentos hacia las escuelas. 
Figura 1 - Formas de contratación permitidas para que las Juntas realicen la compra de alimentos para los comedores estudiantiles.

\section{FORMAS DE CONTRATACIÓN}

¿Hay presencia del CNP en la zona? ¿Hay organizaciones de agricultores vinculadas con en CNP?

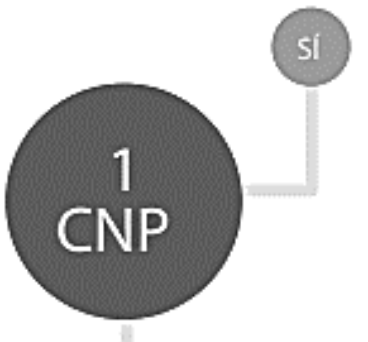

El artículo 9 de la Ley $n^{\circ} 2035$ Ley Orgánica del CNP dice: "Los entes públicos están obligados a proveerse del Consejo Nacional de Producción (CNP) todo tipo de suministros genéricos propios del tráfico de esta institución, a los precios establecidos. Para tal efecto, dichos entes quedan facultados para que contraten esos suministros directamente con el CNP, el cual no podrá delegar ni ceder, en forma alguna esta función"

¿Tiene capacidad de respuesta?

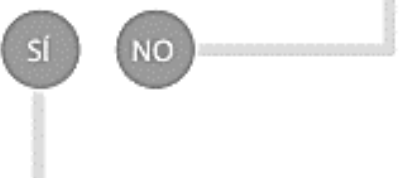

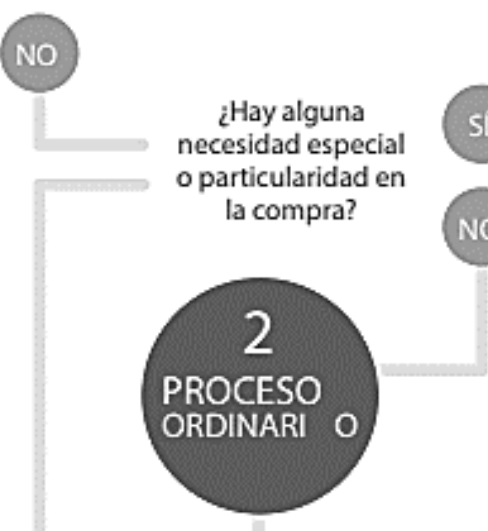

Generar un concurso de contratación de acuerdo con los montos totales de la compra.

Si el CNP no tiene la capacidad para dar respuesta a la demanda, se procede a invitar a concurso para contratación directa Oficio $\mathrm{N}^{\circ} 6571$ (DAGJ-959-2002) de 05 de junio de 2002, faculta recurrir a otros medios legales de contratación para que el servicio no se vea afectado.

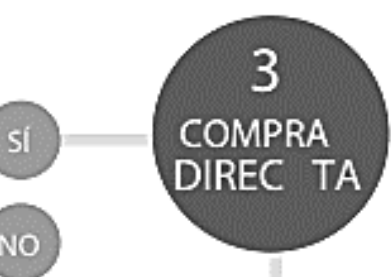

Se invita al menos a tres oferentes y se selecciona la mejor oferta, según las condiciones que haya establecido la junta de educación o administración en el pliego de condiciones.

Por la necesidad y particularidad de la compra, la Contraloría General de la República ha permitido expresamente la compra directa concursada a algunas juntas, independientemente del monto total

Según oficio 13728.

\section{SE REALIZA LA COMPRA}

Fuente: FALLAS; RAMÍREZ; SOLÍs. Proceso de compra de frutas y hortalizas a los agricultores familiares: Guía dirigida a juntas de educación y juntas administrativas de centros educativos. UNA/BRASIL/FAO, 2014

En el caso \#1, la contratación directa la realiza el Consejo Nacional de Producción (CNP) por medio del Programa de Abastecimiento Institucional (PAI). El CNP es la entidad encargada de regular el proceso de compra de alimentos para proveer a las instituciones públicas del país. La Ley Orgánica del CNP, № 2035 de 1956 y su reforma a partir de la Ley 6050 en el año 1977, establece en su Artículo $1^{\circ}$ que "[...] tendrá personalidad jurídica propia y gozará de la autonomía funcional y administrativa consagrada en el artículo 188 de la Constitución Política” (COSTA RICA, 1977). Además, según el artículo $5^{\circ}$ de la misma Ley,

Para el cumplimiento de sus fines, el Consejo tendrá las siguientes atribuciones: a) Comprar directa y exclusivamente a los productores nacionales artículos básicos de consumo popular a precios que garanticen utilidades justas, contribuyendo así a fomentar esa producción. Solamente en casos excepcionales, por acuerdo razonado tomado por 
dos tercios o más del total de miembros de la Junta Directiva, podría comprar a los intermediarios [...] (COSTA RICA, 1977).

Según lo dispuesto en el Art. 11 de dicha Ley, los productos autorizados para abastecer a las instituciones públicas serán de origen nacional. Para el caso de la comercialización de productos procedentes de la importación, sólo se autoriza en caso de que el producto y/o materia prima no sea producido en el país o si existiera desabastecimiento local del producto, según lo dispone el Art. 20 del Reglamento. De esta forma, el CNP es la institución encargada del abastecimiento de productos agrícolas y agroindustriales en los programas y proyectos institucionales del Estado (incluyendo el PANEA), con el propósito de disminuir sus costos de operación y permitir al agricultor/a nacionales tener acceso a ese mercado (CNP, 2017).

Al igual que las Juntas, el proceso para contratación de proveedores del PAI debe sujetarse al cumplimiento de los principios generales dispuestos en la Ley General de Contratación Administrativa $\mathrm{N}^{\circ}$ 7494, en concordancia con el Art. 9 de la Ley Orgánica del CNP. Sin embargo, en el año 2008, el CNP fue cuestionado porque los principales proveedores que formaban parte de su registro para abastecer las instituciones, eran grandes empresas de alimentos. Esta situación generó un debate público debido a las limitadas vías de comercialización directa que existen para los pequeños y medianos productores en el país, las cuales estaban siendo restringidas a las ferias del agricultor. Ante esta situación, en el año 2008, el Artículo 9 vigente en la Ley 2035 del CNP, es reafirmado y fortalecido mediante la Ley 8700 , en la cual se expresa la obligatoriedad de realizar la compra de alimentos a micro, pequeños y medianos productores, como se indica en la Ley 8700:

\begin{abstract}
Artículo 9- Los entes públicos están obligados a proveerse del Consejo Nacional de Producción (CNP) todo tipo de suministros genéricos propios del tráfico de esta institución, a los precios establecidos. El Consejo Nacional de Producción debe fungir, con carácter de prioridad, como facilitador en el acceso al mercado institucional a los micro, pequeños y medianos productores agropecuarios, agroindustriales, pesqueros y acuícola de Costa Rica (COSTA RICA/CNP, 2008).
\end{abstract}

De acuerdo con la Ley, este proceso incluiría la disposición de centros de acopio y transporte de los alimentos hasta su lugar de abastecimiento, facilitando la logística a aquellos productores que no cuentan con dichas capacidades. Para Sáenz et al. (2013) el PAI no era un sistema de compras consolidado, pues aunque con la Ley se obliga a las instituciones públicas a abastecerse de alimentos por medio de esta plataforma de compras, que representa un mercado potencial de 104.000 millones de colones en compras, el CNP apenas tenía una participación activa del $23 \%$. Esto quiere decir, que la mayoría de instituciones públicas tenían otras formas de abastecimiento diferentes al PAI del CNP.

La reformulación del Artículo $9^{\circ}$, generó desafíos importantes para el CNP, cuyos principales problemas eran operativos, pues no tenía la capacidad financiera para manejar el costo que representa el pago durante el tiempo que transcurre entre recibir los pagos de las instituciones que demandan alimentos y pagar a los proveedores, debido a la falta de un colchón financiero que lo permitiera (SÁENZ; CHAVES, 2013). Esta situación y la constante intención de cerrar al CNP durante los dos gobiernos anteriores a la ejecución de los proyectos piloto en estudio (2006-2010 y 2010-2014), afectaron a la institución en términos de cantidad de personal y recursos económicos y materiales para realizar sus operaciones. A su vez, el CNP mantenía como suplidores o proveedores, a empresas que poseían capacidades logísticas para el abastecimiento, sin dar prioridad a productores nacionales de menor escala de producción y dependientes del mercado interno. 
Para el año 2015, el conjunto de todas las instituciones del Estado (Gobierno Central/ Entes descentralizados o autónomos) disponían de 105500 millones de colones para satisfacer la demanda de alimentos (abarrotes, carnes, frutas, hortalizas, verduras, productos básicos, lácteos, huevos, pescado y otros). Ante este panorama, la política del CNP, en apoyo a los productores nacionales, fue ir cubriendo progresivamente este mercado, para garantizarles la comercialización de sus productos en este circuito comercial y promover su gestión competitiva (CNP, 2015b).

De acuerdo al Reglamento del PAI, para aceptar o rechazar proveedores, en el CNP existe una Comisión de Abastecimiento encargada de resolver administrativamente la aprobación o rechazo de los proveedores que cumplen o no con los requisitos establecidos y, que han sido aprobados previamente desde las Oficinas Regionales del CNP. Por otro lado, para aprobar a las empresas grandes como proveedoras del PAI, la Comisión debe presentar un informe trimestral a la Junta Directiva, justificando la compra. Posteriormente éste debe ser avalado por la Contraloría General de la República.

El desafío operativo del CNP para pasar de comprar alimentos de los grandes proveedores a estimular las compras de alimentos producidos por micro, pequeños y medianos productores (serán llamados de agricultores o familias agricultoras), fue considerado como un proceso gradual. Esta situación dio paso a ciertas reflexiones acerca del margen de permisión que tienen las grandes empresas en la esfera pública y con la alimentación.

Una de las razones principales para que existiera la aprobación de los dos mecanismos de abastecimiento mencionados (por medio del CNP y de forma directa), era la falta de capacidades y poca rentabilidad que representaba para el CNP el atender la totalidad de los centros educativos, sobre todo en algunas zonas más alejadas y en centros educativos con menos población infantil.

En el año 2017 la DPE del Ministerio de Educación Pública realizó un trabajo en conjunto con el CNP (Certificación DPE-667-2016 del 7/11/2016) para el fortalecimiento de las proveedurías de los centros educativos. De esa forma, ambas instituciones identificaron los centros educativos que irían a ser atendidos por el CNP (Oficio No 17195 y oficio DCA- 3193 de la División de Contratación Administrativa ), sin embargo, dadas las posibilidades del PAI en ese momento, se indicó:

Que pese a los esfuerzos realizados por ambas instituciones, quedan Juntas de Educación y Administrativas que no podrán ser abastecidas por el CNP el próximo año 2017, por lo que el Ministerio en aras de buscar la mejor alternativa que garantice el derecho a la alimentación de los niños, niñas y adolescentes, en procura del interés público inmerso en el desarrollo del programa de alimentación y nutrición de los centros educativos, ha considerado presentar una nueva solicitud de autorización a nombre de estos centros educativos, para que se les permita contratar en forma directa el abastecimiento de los alimentos para ser preparados en los comedores estudiantiles, para lo cual remite el listado de Juntas que no será atendidas por el CNP en el curso lectivo 2017 (OFICIO DCA3193 División de Contratación Administrativa, diciembre 2016).

Con esas disposiciones, el CNP iría a abastecer alrededor de un 28\% del total de centros educativos. Por su parte, aquellas Juntas que por razones justificadas no podrían abastecerse mediante el PAI, debían seguir el procedimiento de selección de proveedores promoviendo la contratación directa concursada, en la que se inviten al menos tres oferentes, este sería el proceso de contratación directa \#3 de la Figura 1, que corresponde al caso de Frailes de Desamparados. La contratación directa concursada, en vez del seguimiento al procedimiento ordinario que corresponde, fue una decisión tomada por el 
MEP después de haber analizado la conveniencia y oportunidad que tienen las Juntas, como se indica:

No obstante lo anterior, señala el Ministerio que a pesar de los esfuerzos realizados por ambas instituciones, todavía quedan centros educativos que no serán suplidos por el CNP en el próximo curso lectivo 2017, precisamente sobre los cuales se requiere la autorización indicada, pues ha analizado la conveniencia y oportunidad de que las Juntas realicen una contratación directa concursada y no el procedimiento ordinario que corresponde, por el tiempo que lleva promover una licitación, por la lejanía de las Juntas en muchos casos, por los pocos proveedores de alimentos en las zonas y por la sencilla formación académica de los miembros de estas Juntas que les imposibilidad llevar adecuadamente un proceso de licitación, circunstancias que ponen en riesgo la debida satisfacción del interés público y el derecho constitucional a la educación de los niños, niñas y adolescentes, que reciben el beneficio como parte de una educación integral (DIVISIÓN de contratación administrativa, 2016. DCA- 3193).

Durante los años siguientes y hasta la actualidad, el proceso de inclusión de pequeños y medianos productores como proveedores del PAI, continúa siendo un desafío para el cumplimiento de las directrices que se establecen para ejecutar el programa. En ese sentido, identificar las limitaciones y potencialidades principales vividas por los actores durante sus experiencias en ese contexto, proporciona una base de trabajo hacia propuestas que se dirijan a la búsqueda de alternativas que modifiquen o mejoren los mecanismo actuales, Así, buscar formas de abastecimiento de alimentos en los comedores escolares, que se beneficien de la reconexión entre la producción el consumo de alimentos locales.

\section{Principales potencialidades y limitaciones durante el abastecimiento de alimentos producidos por familias agricultoras en la alimentación escolar}

Durante el proceso investigativo los actores involucrados opinaron acerca de diversas acciones que funcionaron y otras que no durante su participación en los proyectos piloto. Aquellos aspectos que los actores mencionaron que no funcionaron, fueron considerados como determinantes que limitaron la efectividad del proceso. Por su parte, aquellos aspectos que los actores indicaron que sí funcionaron durante la implementación de los proyectos piloto, fueron considerados como determinantes potenciales de la misma, proporcionando vías para fortalecer futuras acciones.

\subsection{Las potencialidades}

Una de las potencialidades del proceso de abastecimiento, fue la inclusión de nuevas organizaciones de agricultores como proveedores del PAI, atendiendo a las disposiciones de la Ley $\mathrm{N}^{\circ} 8700^{1}$. Esto permitió el acceso al mercado institucional en lugares en donde no se había contemplado antes, como en el caso del Centro Agrícola Cantonal de Coto Brus. Aunque se requiere de mejoras sustanciales para fortalecer el proceso, la estrategia permitió ampliar las oportunidades de acceso a mercados para algunos

\footnotetext{
1 "El Consejo Nacional de Producción debe fungir, con carácter de prioridad, como facilitador en el acceso al mercado institucional a los micro, pequeños y medianos productores agropecuarios, agroindustriales, pesqueros y acuícola de Costa Rica" (COSTA RICA/CNP, 2008).
} 
agricultores, siendo complementario a la participación en mercados locales de circuito corto $^{2}$ (venta de casa en casa, Feria o venta a comercios locales) que existen en las comunidades. Según Schneider (2016), los mercados institucionales son el resultado de una construcción social y política, que tiene que ver con fondos públicos (SCHNEIDER, 2016). Estos, se colocan como oportunidades, en la medida en que éstos amplíen los horizontes de posibilidades de venta de los alimentos que producen los agricultores (GAZOLLA, 2017).

También se identificó un compromiso en el cumplimiento del proceso de comercialización, tanto por parte de los y las agricultoras hacia los encargados de las organizaciones agrícolas (CACs y cooperativas), como por parte de los dirigentes de dichas organizaciones con el CNP. Inclusive en el caso de Coto Brus, en donde el mercado institucional no fue muy prometedor en términos de rentabilidad económica para los agricultores, estos se mantuvieron como proveedores por el compromiso asumido y porque tenían expectativas de mejores ganancias futuras.

Estas lógicas de participación, en parte pueden ser explicadas a partir de la sociología económica”, en la que se sitúa la discusión sobre la "construcción social de los mercados" (GRAVINA, 2004; PÉREZ-CASSARINO; FERREIRA, 2016). En esta se destaca el papel clave de las relaciones de los actores a partir de redes sociales, como un componente esencial para el funcionamiento de los mercados (WILKINSON, 2010; RADOMSKY, 2006). EI establecimiento de una agencia del CNP en Coto Brus, permitió relaciones más próximas entre actores de la sociedad civil y gubernamentales durante el proceso de abastecimiento, caso contrario se produjo en Frailes, en donde existió menos relación entre los actores institucionales con los actores del CAC para desarrollar los proyectos. Como lo indica Matte et al. (2016), estas relaciones entre los actores sociales, tiene como base "el lugar" en el que ocurre una aproximación de prácticas de producción y consumo. Así, al existir contactos personales entre los actores de las diferentes entidades, se facilitó el proceso de comercialización. Sin embargo, el compromiso de los actores también estuvo determinado por aspectos éticos, que sobrepasa las relaciones sociales y el proceso de transacción económica propias del mercado. Estos se refieren a una racionalidad para actuar de acuerdo a ciertos principios, orientados por el reconocimiento del papel que cumplen como proveedores de alimentos para la población infantil de sus comunidades. Como lo indica uno de los actores del CAC:

Lo importante es llevar los productos frescos de buena calidad y que no tienen un alto contenido de químicos, ya que los productores también somos padres de familia. Lo que queremos es el bienestar de los jóvenes que consumen los productos, que tengan una mejor calidad de vida (actor: CACFG1, 2017).

Por otro lado, dentro de las potencialidades de del proceso de abastecimiento, el CNP tiene la capacidad de mantener un registro de información sobre la demanda de alimentos que realizan las Juntas Escolares. Esto representa un proceso de control de los recursos públicos que se destinan a las Juntas, permitiendo identificar los tipos de

\footnotetext{
${ }^{2}$ Una de las características más importantes de un circuito corto o cadena corta es el hecho de que un producto llega a las manos del consumidor con informaciones que le permiten saber dónde fue producido el (lugar), por quien (productor) y de qué forma (sistema de producción) (MARSDEN et al., 2000).

${ }^{3}$ La sociología económica es identificada con los trabajos de Mark Granovetter a partir de los años 70 en su tesis de doctorado, Geting a Job (1974), estableció las ambiciones del enfoque así como su metodología: el estudio de las dinámicas de los mercados vistos como extrapolaciones de redes sociales.
} 
alimentos que se solicitan y la inversión que realizan estas entidades durante el pago del pedido. Este determinante tiene importantes implicaciones para el control y monitoreo de los recursos de FODESAF y del MEP que se gira a dichas instancias, pues los mecanismos de control de dichas instancias en ese sentido, son escasas o inexistentes. Sobre el tema del control y monitoreo de recursos, es una de las deficiencias que Cárdenas (2013) había identificado durante lo que llamó, la fase de estancamiento del PANEA, indicando que es un periodo que comenzó a partir del año 2000.

Otra potencialidad del proceso, fue la capacidad de autogestión en la organización que tuvieron los presidentes de los CACs de Frailes y de Coto Brus al liderar los procesos de abastecimiento. Esta situación permitió que sus organizaciones se convirtieran en proveedores directos de los centros educativos (en el caso de Frailes) o del PAI (en el caso de Coto Brus). Pese a dificultades relacionadas con la producción de alimentos como la diversificación, falta de asistencia técnica e insumos para la producción, entre otros, los actores de los CACs asumieron su papel de proveedores, haciendo uso de sus capacidades organizacionales, para convocar e instaurar el proceso que implicaba el acopio, la comercialización y entrega de los alimentos en los centros educativos.

Un aspecto que favoreció la disponibilidad de alimentos en el nivel local, fue la existencia de mercados locales de circuito corto $^{4}$, en donde los agricultores comercializaban los alimentos. Las familias que contaban con estas oportunidades alternativas de mercado, lograron mantenerse como proveedoras para el abastecimiento institucional con mayor facilidad que aquellas que no contaban con otras alternativas de mercado. De acuerdo con los actores, su participación en mercados como la Feria Cotobruseña (en Coto Brus), y la venta de casa en casa o en locales comerciales de sus comunidades (en Frailes y en Coto Brus), les proporciona una entrada económica y disminuye la incertidumbre ante el hecho de tener que depender de un único mercado (mercado institucional).

Otro aspecto que favoreció el proceso de abastecimiento fue la asesoría técnica dirigidas a las y los agricultores en temas de comercialización y mercadeo de alimentos, la cual fue realizada por algunos actores del sector académico (de Universidades estatales: UNED, TEC y UCR), con el fin de mejorar el ingreso económico para las familias. Sin embargo, al igual que los procesos de capacitación y asesoría técnica para la producción, es necesario que este fortalecimiento de capacidades sea permanente, requiriéndose de programas y acciones estatales permanentes en este ámbito.

Los CACs (Frailes y Coto Brus) cumplieron una función importante como instancia intermediaria entre familias agricultoras y escuelas (caso Frailes) o entre las familias agricultoras y CNP (caso Coto Brus), pues les aseguraron el pago al contado y oportuno. Sin embargo, esta situación varía en cuanto a su permanencia en el tiempo. Por ejemplo, en el caso del CAC de Frailes, se trata de una entidad que cuenta con un capital más robusto para enfrentar esa necesidad. Sin embargo, en el caso del CAC de Coto Brus, esta situación comprometió el capital y funcionamiento para atenderlo de forma sostenida. De acuerdo con los actores, el trabajo que realiza el presidente de este CAC es ad honorem y, en el caso del asistente administrativo, quien también es agricultor, este recibe un salario inferior al salario mínimo. Cabe señalar que a pesar de esa situación, estas personas mantienen un compromiso con el proceso, principalmente por lo que representa para sus vecinos agricultores, así como por sus expectativas de mejora.

Otro aspecto que se considera potencial tiene que ver con las relaciones de amistad establecidas entre los actores de las organizaciones de agricultores y de los centros educativos durante el abastecimiento de alimentos. Este tipo de relaciones entre los actores, permitió la flexibilidad por parte de las organizaciones de agricultores, para realizar

\footnotetext{
${ }^{4}$ circuitos cortos de comercialización, es decir, en donde se da una venta directa entre el productor y el consumidor, o bien vía un único intermediario (NIEDERLE; ALMEIA; VEZZANI, 2013).
} 
cambios en los alimentos que no cumplían con las expectativas de los encargados del recibo de alimentos en los centros educativos. Estas manifestaciones permiten comprender que el proceso de abastecimiento institucional, no se trata de una simple relación de mercado pautada por aspectos económicos exclusivos de la comercialización, sino que median aspectos éticos y socioculturales para favorecerlo.

Las relaciones próximas entre quien produce y quien consume permitieron la entrega de alimentos frescos, que habían sido cosechados el mismo día o el día anterior, lo que asegura una mejor calidad nutricional del alimento. Esto fue posible tanto por la cercanía de los agricultores a los CACs, como la cercanía de estas organizaciones con los centros educativos. Las relaciones establecidas entre las personas, le dan un carácter social y ético al proceso de abastecimiento. Según Pilla (2005), las escogencias de alimentos traen consigo la marca cultural del medio en el cual se desarrollaron. En ese sentido, las cocineras de los centros educativos indicaron:

Para mí, en lo personal, lo importante es más que todo recibir un producto de buena calidad. Le puedo decir a CACFG5: "Necesito que me traiga 15 chayotes" y yo sé que CACFG 5 va y prácticamente los toma de la planta y me los trae. Igual con el culantro, ellos lo traen "verdecitico" (muy verde), recién arrancado (Actor: EscSE2, 2017).

La entrega de alimentos frescos que realizan los CACs (tanto en Frailes como en Coto Brus) hacia los centros educativos, además de ser un determinante del consumo también es determinante de la utilización biológica del alimento. De acuerdo con los actores, aunque existen parámetros de calidad propuestos por el CNP, la entrega de alimentos en el centro escolar, depende de las expectativas de calidad de las personas que reciben el pedido en el centro educativo.

En el caso de las señoras del comedor que reciben el pedido, estas indicaron que sus expectativas de calidad de los alimentos entregados por los CACs eran satisfactorias, principalmente por tratarse de alimentos frescos. Esa percepción tiene que ver con una dimensión relacional de la calidad, la cual permite dar validez y reconocimiento a las características de frescura del alimento y se puede deber a que la relación directa actúa como un mecanismo de juicio de la calidad de los alimentos, al permitir conocer al productor y el lugar de donde vienen los alimentos que produce, siendo que para el caso de las personas del centro educativo, el recibir alimento de los CACs, tiene que ver también con alimentos cultivados por familias que son sus vecinos en la comunidad.

De acuerdo con Prigent-Simonin et al. (2005), la dimensión relacional de la calidad, tiene que ver con el papel que juega la confianza (credibilidad, integridad y benevolencia) en la percepción de la calidad de los alimentos, la cual se establece a partir de las relaciones entre productores y consumidores. Según las autoras, el tipo de relación define la dimensión de la calidad que establece la venta del alimento y, para el caso en que existen relacionamiento intensos, es decir en donde productor y consumidor se reúnen directamente para entregar el alimento, como en este caso, la dimensión emocional combina conocimientos y creencias que determinan la aceptación. Para representar este tema, una de las cocineras en un centro educativo hizo una comparación de proveedores de otras zonas y los actuales proveedores que corresponden al CAC:

Venían desde Pérez Zeledón con las papayas, con el culantro, con las lechugas, sin un carro de frío, que no tenía cajón, con unas condiciones inapropiadas y aquí me llegaron con todo ese pedido hecho un desastre. El camión se les estropeó, todos los productos durmieron esa noche en el cajón y llegaron hasta el día siguiente. Cuando usted recibe una mercadería así y cuando usted se da cuenta que eso sucede, usted se da cuenta de que eso es algo muy absurdo. Todos dijimos ¿Por qué eso tan 
absurdo? ¿Por qué si tenemos el Centro Agrícola Cantonal aquí cerca, donde a usted le traen todos los productos frescos y de buena calidad, ¿Por qué el PAl tiene que comprarle a alguien que ni siquiera es del cantón? Entonces ellos (CNP) vinieron e hicieron una distribución de toda la ruta $y$, si no me equivoco, ellos mismos desestimaron, porque vieron que era demasiado gasto, absurdo el trayecto y demasiado costo para venir a entregar. Entonces nosotros, en buena hora, de nuevo, nos pusimos a coordinar con el Centro Agrícola Cantonal (Actor: EscSE1, 2017).

Como se mencionó enl apartados anteriores, según las señoras del comedor y las personas encargadas de recibo de alimentos en los centros educativos, la entrega de alimentos por parte de los CACs representó un beneficio en térmicos de calidad, frescura y flexibilidad para cambio o solicitud de alimentos que no cumplían con sus expectativas cómo clientes. Sin embargo, esta situación no ocurre con la entrega realizada por otros proveedores que son de otras localidades. En estos casos, existieron limitaciones porque la calidad de los alimentos no cumplía con las expectativas de los miembros de los centros educativos. En este aspecto, puede influir el hecho de que, personas diferentes realizan la entrega y no existen procesos sociales consolidados, como en el caso de los CACs. Al respecto, Murdoch y Miele (2013) argumentan que cuando los alimentos pasan por un proceso de calificación, la calidad es capaz de ser alterada por la intervención social, proceso que es difícil a una escala industrial en donde los productos son altamente uniformes. Por tanto, en el proceso de entrega de alimentos en el centro educativo, cabe valorar si los parámetros institucionales son suficientes e incluyen aspectos que valoricen el papel de consumidor-ciudadano, por encima de un consumidor-cliente.

\subsection{Limitaciones}

Una de las principales limitaciones del abastecimiento es el no contar con un sistema nacional de información sobre la oferta de alimentos, lo cual se relaciona con las deficientes capacidades institucionales para establecer mecanismos de registro y actualización sobre información agrícola en el país. De acuerdo con los actores gubernamentales, este problema se complica porque la mayoría de agricultores del país no pertenecen a organizaciones. Según el Censo Nacional Agropecuario (2014), del total de productores costarricenses ( 80 987), solamente un 29,1\% pertenece a alguna organización (17,2\% cooperativa, 9,3\% asociación o cámara y 3,2\% a un sindicato), situación que dificulta el poder mantener registros actualizados. De acuerdo con el caso brasileiro, algunos autores han considerado la falta de registros actualizados, como uno de los principales obstáculos para operacionalizar el proceso de abastecimiento (FERNANDES; SCHNEIDER; TRICHES, 2016).

Un aspecto que solventa en alguna medida la deficiente información sobre oferta de alimentos, es el hecho de que los CACs cuentan con registros sobre las características de producción y de los agricultores asociados. Sin embargo, esto no es suficiente para solucionar la falta de registro a nivel nacional. Según Fernandes et al. (2016), las dificultades en la obtención de este tipo de datos oficiales nacionales, es uno de los problemas mayores que imposibilita el realizar conexiones entre la producción y el consumo de alimentos.

Por su parte, a pesar de existir información de la demanda de alimentos que realizan las Juntas escolares al CNP, una de las limitaciones que perjudican el proceso es el desconocimiento acerca de esa demanda de alimentos, por parte de los agricultores. Ese flujo de información permitiría a las familias agricultoras, planificar su producción en función de los alimentos necesarios. Desde esta perspectiva, el menú es una herramienta capaz de establecer interrelaciones desde la producción hacia el consumo y desde el consumo hacia la producción de alimentos, siendo clave para la planificación y organización 
de la producción. De ahí la importancia de contemplar durante la elaboración del menú, tanto la producción local como las necesidades nutricionales que tienen los escolares.

Por otro lado, a pesar de la ventaja que representa para las familias agricultoras el hecho de participar en los mercados locales como formas complementarias al proceso de abastecimiento, los entrevistados mencionaron que, tanto en esos mercados como en el Programa de Abastecimiento Institucional, existe un acaparamiento de mercados por parte de sectores dominantes ${ }^{5}$. Esta es una situación a nivel nacional que tiene que ver con la expansión, crecimiento y concentración empresarial, lo cual ha limitado los canales de comercialización y estabilidad en la venta de alimentos producidos a nivel local por familias agricultoras. Por su parte, el oportunismo de negociantes que acaparan los mercados obstaculiza la expresión y valor que tienen los alimentos locales en el contexto comunitario, y la justicia social.

La problemática mencionada responde al dominio desde la esfera económica, en donde la cultura de producirse, distribuirse y consumirse aquello que es considerado como más rentable, responde a esa lógica comercial y a los intereses económicos de grandes empresas (ESTEVE, 2017). La situación no es tan diferente para el resto de países de América Latina; sin embargo, el crecimiento acelerado de este tipo de empresas en el territorio costarricense, ha dejado en evidencia los problemas sobre el acceso a alimentos producidos por las familias agricultoras.

En ese sentido, Triches; Bacarin (2016 p. 91) indican que el caso de las compras públicas a la agricultura familiar, merece reflexión en lo concerniente al margen que existe a actitudes oportunistas, de empresas no vinculadas a los agricultores familiares, y el permiso que tienen estos para participar, obteniendo ventajas en la comercialización. Esta situación está relacionada con el compromiso gubernamental por hacer efectiva la Ley $\mathrm{N}^{\circ} 8700^{6}$. A su vez, tiene relación con la ética en la que se encuentra sustentado el compromiso gubernamental para decidir sobre el apoyo a las familias agricultoras, por medio de oportunidades de mercado que han sido destinados para ellas.

Otra limitación que afectó la cantidad de alimentos disponibles para el abastecimiento, fue la falta de condiciones de infraestructura adecuada para el acopio de alimentos en las organizaciones de agricultores proveedoras. Este es el caso del CAC de Frailes, en donde según los actores entrevistados, tenían altas expectativas de participación como proveedores del PAI por medio de su participación en los proyectos piloto de la iniciativa Escuelas Sostenibles. Pensaban además, que el hecho de ser parte del PAl los incluiría en un mercado mayor que contemplaba varios centros educativos de la localidad. Sin embargo, a pesar de su motivación y asistencia a reuniones y un curso de capacitación sobre el tema, solamente dos familias agricultoras lograron proveer al CAC. Dos de las razones principales para que esto sucediera fue la presencia del PAI en la zona abasteciendo a los centros educativos por medio de alimentos de otras regiones, dadas las condiciones del CAC, el cual no contaba con los requisitos para ser proveedor del CNP. Otro aspecto importante fue que las dos familias agricultoras que lograron abastecer al CAC para la comercialización directa hacia los únicos dos centros educativos que no estaban siendo atendidos por el PAl, tenían experiencia en comercialización con otros mercados.

Si bien es cierto, se iniciaron los procesos de solicitud al Instituto Nacional de Desarrollo Rural (INDER) para obtener financiamiento que le permitiera al CAC construir instalaciones apropiadas, los procesos burocráticos fueron ineficientes y al momento de

\footnotetext{
${ }^{5}$ Éstos se refieren a sectores que realizan actividades de producción, distribución y venta de alimentos en grandes escalas, perteneciendo a circuitos largos de comercialización

${ }^{6}$ Ley Orgánica del CNP y su artículo 9 en relación con la prioridad a micro, pequeños y medianos productores como proveedores del PAI.
} 
esta investigación, ya habían transcurrido dos años de no obtener respuesta. Como lo indicó uno de los actores del CAC de Frailes:

\begin{abstract}
Hemos hecho muchas acciones administrativas para tratar de conseguir el centro de acopio, en lo que más hemos ido trabajando es para lograr eso. Han durado muchísimo, por la burocracia y todo el entrabamiento que hay a nivel del gobierno en el país. Hemos luchado mucho y hablado mucho sobre eso porque a nivel regional, llega a poner muchas trabas. Por ejemplo, con el control de permisos de la municipalidad (actor: CACFG4, 2017).
\end{abstract}

En este caso, de acuerdo con Sen $\left(2010^{a}\right.$, p. 21$)$ el propio hecho de negar el acceso a los mercados de productos, es una de las privaciones más frecuentes que sufren los pequeños agricultores; además, la libertad de participar del intercambio económico tiene un papel básico en la vida social (SEN, 2010 a p.21). De esta forma, las transacciones, mercados y privaciones de libertad económica para este grupo de agricultores, de acuerdo con los actores locales, han sido limitados por la discordancia entre las disposiciones existentes en la Ley del CNP, la burocracia institucional que no resuelve de forma oportuna y las expectativas de participación generadas por la iniciativa Escuelas Sostenibles.

Otro aspecto que perjudicó el proceso fue la inadecuada manera de solicitud de alimentos, realizada por el CNP al CAC de Coto Brus, para abastecer a los centros educativos; la misma, no correspondía con la capacidad de comercialización de dicha organización. En este caso el CAC se vio obligado a comprar abarrotes u otros alimentos que tenían que conseguirse en otros cantones de la Región. La razón principal de solicitar alimentos o productos que no tenían que ver con la oferta del CAC, respondía a las facilidades logísticas que representaba para el centro educativo y para el CNP, el contar con un único proveedor para suplir la totalidad de alimentos pedidos. Esta situación se relaciona con la dimensión política, siendo requerida una mejor planificación intersectorial para la concertación local de acciones que beneficie a productores y consumidores durante el abastecimiento. Así como el contemplar en esa planificación, un proceso diferenciado para abrir oportunidades para la agricultura familiar.

Por otro lado, la compra de alimentos que el CNP realizó a las organizaciones de agricultores en el caso de Coto Brus (CAC y cooperativas) para abastecer las escuelas, no fue tan favorable como el trato que hubo entre los agricultores con los CACs. Una de las limitaciones más importantes fue el precio establecido por el CNP para pagar los alimentos. De acuerdo con los actores de las organizaciones de agricultores, los precios establecidos no correspondían con las características y costo real de los alimentos a nivel local o regional. Esta situación creó un malestar compartido entre las organizaciones.

Por su parte, en el caso del CAC de Coto Brus, el hecho de tener que comprar varios alimentos fuera de la zona o abarrotes que no eran parte de su oferta, aumentó los costos de transporte y pago a intermediarios para la obtención de dichos alimentos. Estos costos no fueron contemplados en el precio fijado por el CNP durante la compra de los mismos para el PAI, resultando en una venta poco rentable. La razón principal es que el CNP, no estableció diferencias de precio local que contemplaran situaciones de producción o transporte en casos particulares, sino que el precio de alimentos es centralizado y establecido a nivel nacional.

A pesar de los problemas de precio bajo en algunos de los alimentos, existieron compensaciones en el pago por otros alimentos, permitiendo el mantenimiento de las organizaciones de agricultores como proveedoras del PAI. Esta situación es similar a lo que le sucede a las organizaciones de agricultores en Brasil. De acuerdo con un estudio realizado por Lozano, Barreto y Dias (2016) en la cooperativa COMAFITT, los principios de redistribución es un brillante artificio que elimina la especulación, y más importante aún, es 
una transacción justa desde la perspectiva de los agricultores que tiene que ver con la dimensión económica y política.

Otro aspecto que limitó el proceso de abastecimiento fue la falta de información sobre el mecanismo que utiliza el CNP para el establecimiento de precios que realiza el CNP durante la compra de alimentos destinados al PAI. Esta situación, aunado a que existe un porcentaje destinado a los costos de operación del CNP para la gestión del programa, creó un ambiente de desconfianza que afectó el proceso de comercialización. De acuerdo con el Sector Agroalimentario y el CNP (2017), el procedimiento para establecer el precio de los alimentos que se compran a los agricultores, se realiza contemplando el precio máximo de tres plazas por semana de los precios mayoristas en el CENADA ${ }^{7}$ (SECTOR AGROALIMENTARIO y CNP, 2017). Sobre el porcentaje que cobra el CNP por los costos de operación del PAI, se generó una situación que no favoreció el proceso, como lo comenta el siguiente actor:

Con la parte de los centros educativos ha habido un choque de conflictos por los directores. En el sentido que los centros educativos han estado disconformes, porque hay una comisión que se les cobra y que a veces dicen que no es justo, y ese porcentaje del CNP (5\%) les afecta (actor: Coopesab1, 2017).

Otra de las limitaciones principales tuvo que ver con la participación de los actores y su condición como agentes en la construcción de sus procesos de mejora. Muchas veces, no existe ese margen de participación para que los actores sean parte de la toma de decisiones y planificación de proyectos que afectan su quehacer. Esta situación ocurrió en el caso de Coto Brus, en donde los actores del CAC mencionaron que una de sus expectativas era, poder desarrollar un sello de certificación participativa para diferenciar los alimentos que comercializan. Sin embargo, estas acciones no fueron contempladas como prioritarias dentro del trabajo realizado por la red de actores que formaron parte del proyecto piloto. De acuerdo con los agricultores y agricultoras, este sello les hubiese permitido posicionar sus alimentos y aprovechar esa ventaja distintiva en el PAl y otros mercados de la zona.

En cuanto al tema del control y monitoreo de la calidad de los alimentos, el CNP cuenta con normas y un departamento especializado para la supervisión de la calidad de la inocuidad alimentaria de sus proveedores. Sin embargo, en la institución hay poco recurso humano para realizar inspecciones periódicas sobre el proceso de entrega de alimentos en los centros educativos. Esta situación afecta considerablemente la confianza de los consumidores, y demuestra la necesidad de la presencia estatal en la preocupación de lo público.

El debilitamiento del CNP en los últimos años, producto de las políticas neoliberales, ha dejado en desventaja su funcionalidad. Aunque en la institución se cuenta con normativas y lineamientos especializados para realizar una inspección de la calidad de la entrega efectiva, la falta de personal para que ésta sea posible, ha generado consecuencias que limitan su distinción social. El poco relacionamiento entre los encargados del PAI con los consumidores en los centros educativos, limitan el establecimiento de acciones de satisfacción del cliente al convertirse en una entrega y un trámite que se limita a lo comercial. Este y otros aspectos mencionados, proyectan desafíos importantes en relación con el deber de las instituciones públicas para hacer efectivo el derecho humano a una alimentación adecuada, así como la claridad de su responsabilidad institucional ante el tema alimentario y la promoción y creación de sistemas alimentarios más sostenibles.

\footnotetext{
${ }^{7}$ Centro Nacional de Abastecimiento y Distribución de Alimentos (CENADA) es el primer Mercado Mayorista de Costa Rica el cual tiene como el abastecimiento de productos perecederos al por mayor para su posterior distribución a los mercados paralelos del país. Disponible en: http://www.pima.go.cr/cenada/. Acceso en: 15 febrero 2017.
} 


\section{Consideraciones finales}

En Costa Rica, tanto el Programa de Alimentación y Nutrición del Escolar y del Adolescente (PANEA) cómo el Programa de Abastecimiento Institucional (PAI) del CNP, constituyen acciones importantes para la seguridad alimentaria y nutricional de grupos vulnerables que forman parte de su población meta. Por un lado, los agricultores familiares sufren las consecuencias de un sistema que no favorece sus economías locales y que tiene repercusiones en su calidad de vida. $Y$ por otro lado, los escolares, quienes requieren de una atención alimentaria y nutricional ante la malnutrición que ha sido considerada como un problema de salud pública y que se refleja en los problemas actuales de obesidad infantil.

Sin embargo, a pesar de la importancia y amplia trayectoria que tienen estos programas desde su creación hasta la actualidad, existen vacíos operacionales que limitan el cumplimiento efectivo de sus objetivos. El artículo evidenció que las principales limitaciones radican en la falta de mecanismos que proporcionen un trato diferenciado de abastecimiento de alimentos producidos por familias agricultoras locales, constituyendo desafíos futuros a la hora de pensar en estrategias de desarrollo humano y sostenible. Por eso, es necesario el compromiso gubernamental para establecer mecanismos permanentes de control y monitoreo que aseguren el cumplimiento de la inserción de alimentos producidos por familias agricultoras en la alimentación escolar, correspondiendo con las disposiciones de la Ley Orgánica del CNP y asegurando la adecuada disposición de alimentos frescos y nutritivos para los escolares. A la vez, se requieren de procesos de retroalimentación que logren la creación de nuevas o el cumplimiento de los actuales lineamientos del PAl, para permitir reconexión entre la producción y el consumo de alimentos locales y con esto, obtener los beneficios que conlleva el contar con sistemas alimentarios más sostenibles.

Es por esa razón, que las potencialidades como resultado de este artículo se consideran una base para la toma de decisiones que impliquen la articulación intersectorial y la participación de los actores involucrados hacia el favorecimiento de procesos de abastecimiento de alimentos producidos por familias agricultoras en la alimentación escolar. El poder analizar las experiencias de los proyectos piloto brinda información sobre aspectos potenciales del proceso, expresados en valores que sobrepasan los aspectos económicos y que se presentan como manifestaciones socioculturales y éticas generadas durante el recibimiento de alimentos en los centros educativos, respondiendo a la construcción social de mercados que caracteriza a las compras públicas de alimentos. Por su parte, el promover la reconexión entre la producción y consumo de alimentos en el nivel local permite asegurar el acceso a alimentos más frescos, característica que asegura un alimento más nutritivo y con calidad sensorial y social más representativa en este tipo de alimentos, cuyo origen es conocido y forma parte de la cultura de los pueblos.

\section{REFERÊNCIAS}

BALEM, T. A.; SCHNEIDER, S.; FIALHO, M. A. V. O lugar dos programas de alimentação escolar na construção da nova equação alimentar. Anais de $53^{\circ}$ Congresso da Sociedade Brasileira de Economia Administração e Sociologia Rural. Agropecuária, Meio Ambiente e Desenvolvimento, João Pessoa. 2015.

\section{CÁRDENAS, A. Los programas de alimentación escolar (PAE) en América Latina:} una propuesta de modernización para Costa Rica. 2013. Tesis (Doctorado em Educación) - Universidad Estatal a Distancia de Costa Rica. San José, 2013. 
CONSEJO NACIONAL DE PRODUCCIÓN. Mercado Institucional: Información general sobre el Programa de Abastecimiento Institucional (PAI) y requisitos para suplidores. Documento técnico en formato de Broshure. Sector Agroalimentario. Costa Rica, 2015b

CONSEJO NACIONAL DE PRODUCCIÓN - CNP. Ministerio de Agricultura y Ganadería. Página web. 2017. Disponible en: https://www.cnp.go.cr/ Acceso: abril. 2020.

COSTA RICA. Ley Orgánica del Consejo Nacional de Producción Nº 6050, 1977

COSTA RICA. Ley Orgánica del Consejo Nacional de Producción № 8700. 2008.

COSTA RICA. Ministerio de Educación Pública. Documento Técnico del PANEA. San José, 2013b. Disponible en:

http://fodesaf.go.cr/prog_soc_selectivos/programacion_anual/fichas_tecnicas/docu mentos/PANEA.pdf Acceso: abr. 2020.

ESTEVE, V. E. O negócio da comida. Quem controla nossa alimentação? 1 ed. Expressão Popular, São Paulo, 2017.

FALLAS, E.; RAMÍREZ, G.; SOLÍS, J. Proceso de compra de frutas y hortalizas a los agricultores familiares: Guía dirigida a juntas de educación y juntas administrativas de centros educativos. UNA/BRASIL/FAO, 2014.

FERNANDES, P.; SCHNEIDER, S.; TRICHES, R. Identificação da oferta de produtos da agricultura familiar e da demanda pelo Programa de Abastecimento Escolar das escolas estaduais do Rio Grande do Sul. En: TEO, R. P. A.; TRICHES, R. M. (orgs.) Alimentação Escolar: construindo interfaces entre saúde, educação e desenvolvimento. Chapecó: Argos, 2016.

GAZOLLA, M. Cadeias Curtas agroalimentares na agroindústria familiar: dinâmicas e atores sociais envolvidos. En: SCHNEIDER, S.; GAZOLLA, M. Cadeias Curtas e redes agroalimentares alternativas: negócios e mercados da agricultura familiar. Porto Alegre: Editora da UFRGS, 2017.

GRAVINA, H. Democratizando el mercado agrícola: mercados locales y participación social. In: CANUTO, J. C: COSTABEBER, J. A (Org.). Agroecologia: conquistando a soberania alimentar. Porto Alegre: Emater/RS-Ascar; Pelotas; Embrapa, 2004.

LOZANO, BARRETO; DIAS (2016). Produzindo e fornecendo alimentos para un cardapio sustentável o caso da COMAFITT. In: TOMÉ, F.; MATTE, A.; SCHNEIDER, S. (orgs.). Produção, consumo e abastecimento de alimentos: desafios e novas estratégias. Porto Alegre: Editora da UFRGS, 2016.

MATTE, A.; SILVA, M. F.; ZAMBONI, M.; WAQUIL, P. D.; SCHNEIDER, S. A construção social dos mercados da pecuária familiar no Sul do Rios Grande do Sul: o caso do 
cordeiro do território Alto Camaquã. En: TOMÉ, F.; MATTE, A.; SCHNEIDER, S. (orgs.). Produção, consumo e abastecimento de alimentos: desafios e novas estratégias. Porto Alegre: Editora da UFRGS, 2016.

MARSDEN, T.; BANKS, J.; BRISTOW, G. Food suply chain approaches: exploring their role in rural development. Sociologia Ruralis, v. 40, n. 4, pp. 424-438, 2000.

Ministerio de Educación Pública (MEP). Lineamientos de Programas de Equidad. Dirección de Programas de Equidad. San José, Costa Rica: Condor Editores, 2012.

MORGAN, K. School Food and the Public Domain: The Politics of the Public Plate. The Political Quarterly, v. 77, n. 3, 2006.

SONNINO, L; BASSINELLO, T. L.; LOZANO, C. School Feeding Programmes: addressing the challenges of sustainable development. In: PÉREZ-CASSARINO, J.; TRICHES, R. M.; BACCARIN, J. G.; TEO, C. R. P. A. (orgs.). Abastecimento Alimentar, Redes Alternativas e Mercados Institucionais. Chapecó: Ed. UFFS, 2018.

MURDOCH, J.; MIELE, M. A new aesthetic of food? Relational reflexivity in the 'alternative' food movement. In: HARVEY, M.; MCMEEKIN, A.; WARDE, A. Qualities of Food. Manchester: Manchester University Press. 2013.

NATIONAL FARM TO SCHOOL NETWORK. Tides Center. Página oficial. 2016. disponible en: http://www.farmtoschool.org/resources-main/growth-of-farm-toschool-in-the-u-s-1997-2014-graphic Acceso: abril, 2020.

NIEDERLE, P.; ALMEIDA, L; VEZZANI, F. M. Agroecologia: práticas, mercados e políticas para uma nova agricultura. Curitiba: Kairós, 2013.

PÉREZ-CASSARINO ,J.; FERREIRA, A. D. D. Redesenhando os mercados: a proposta dos circuitos de proximidade. Espacio Regional. Vol 1, n. ${ }^{\circ}$ 13, Osorno. pag 49-65, 2016.

POWYS PUBLIC PROCUREMENT PARTNERSHIP. Local and local organic food in schools and hospitals contribuiting to the health of our nation. 2002. Disponible en: http://orgprints.org/10844/1/Powys_public_procurement_partnership.pdf Acceso: abril, 2020.

PRIGENT-SIMONIN, A. H; HÉRAULT-FOURNIER, C. The role of trust in the perception of the quality of local food products: with particular reference to direct relationships between producer and consumer. Anthropology of food. 2005. Disponible en: http://aof.revues.org/document204.htm Acceso en: abr. 2020.

RADOMSKY, Guilherme Francisco Waterloo. Reciprocidade, redes sociais e desenvolvimento rural. En: SCHNEIDER, Sergio (Org.). A diversidade da agricultura familiar. Porto Alegre: Ed. da Universidade (UFRGS), p. 104-133, 2006. 
SÁENZ, F.; CHAVES, J. Vigésimo Informe Estado de la Nación en Desarrollo Humano Sostenible. Informe Final. La institucionalidad del Sector Agropecuario

Costarricense: evolución y efectos sobre el desarrollo del sector. San José, 2013.

SCHNEIDER, S. Mercados e Agricultura Familiar. En: MARQUES, F. C.; CONTERATO, M.; SCHNEIDER, S. (orgs.). Construção de Mercados e Agricultura Familiar: Desafios para o Desenvolvimento Rural. Porto Alegre: Editora da UFRGS, 2016.

SEN, A. Desenvolvimento como Liberdade. Tradução Laura Teixeira Mota; revisão técnica Ricardo Doninelli Mendes. São Paulo: Companhia de Letras, 2010a.

TRICHES R. M, BACCARIN J. G. Interações entre alimentação escolar e agricultura familiar para o desenvolvimento local. En: Teo CRPA; Triches, R. M. (Org.).

Alimentação Escolar: construindo interfaces entre saúde, educação e desenvolvimento. 1ed.Chapecó: Argos, 2016, v. 1, p. 89-109.

WILKINSON, J. Mercados, redes e valores: o novo mundo da agricultura familiar. Porto Alegre: UFRGS, 2010.

Marianela Zúñiga-Escobar. Doctora. Docente e investigadora en la Sección de Nutrición Pública de la Escuela de Nutrición, Facultad de Medicina de la Universidad de Costa Rica. marianela.zunigaescobar@ucr.ac.cr

Cátia Grisa. Doctora. Docente en los Programa de Pós-graduação em Desenvolvimento Rural e Programa de Pós-Graduação em Dinâmicas Regionais e Desenvolvimento, Universidade Federal de Rio Grande do Sul - Porto Alegre - Rio Grande do Sul - Brasil. catiagrisaufrgs@gmail.com

Gabriela Coelho-de-Souza. Doctora. Docente en los Programa de Pós-graduação em Desenvolvimento Rural, Universidade Federal de Rio Grande do Sul - Porto Alegre - Rio Grande do Sul - Brasil.gabrielacoelho.ufrgs@gmail.com

Como citar: ZÚÑIGA-ESCOBAR, Marianela; GRISA, Cátia; COELHO-DE-SOUZA, Gabriela. Abastecimiento de alimentos producidos por la agricultura familiar en la alimentación escolar en Costa Rica: Potencialidades y limitaciones. Redes (St. Cruz Sul, Online), Santa Cruz do Sul, v. 25, n. 2, p. 461-481, may 2020. ISSN 1982-6745. Disponível em: https://doi.org/10.17058/redes.v25i2.15086 


\section{CONTRIBUIÇÃO DE CADA AUTOR}

a. Fundamentação teórico-conceitual e problematização: Marianela Zúñiga-Escobar, Cátia Grisa, Gabriela Coelho-de-Souza

b. Pesquisa de dados e análise estatística: Marianela Zúñiga-Escobar

c. Elaboração de figuras e tabelas: Marianela Zúñiga-Escobar

d. Fotos:

e. Elaboração e redação do texto: Marianela Zúñiga-Escobar, Cátia Grisa, Gabriela Coelhode-Souza

f. Seleção das referências bibliográficas: Marianela Zúñiga-Escobar, Cátia Grisa, Gabriela Coelho-de-Souza

Fontes de financiamento: Coordenação de Aperfeiçoamento de Pessoal de Nível Superior (CAPES) y Oficina de Asuntos Internacionales (OAICE) de la Universidad de Costa Rica (UCR). 